\title{
Skimming-trajectory effect for energy loss of medium-energy He ions passing along major crystal axes of $\mathrm{KI}(001)$ and $\mathrm{RbI}(001)$
}

\author{
K. Mitsuhara, ${ }^{1}$ T. Matsuda, ${ }^{1}$ K. Tominaga, ${ }^{1}$ P. L. Grande, ${ }^{2}$ G. Schiwietz,${ }^{3}$ and Y. Kido ${ }^{1, *}$ \\ ${ }^{1}$ Department of Physics, Ritsumeikan University, Kusatsu, Shiga-ken 525-8577, Japan \\ ${ }^{2}$ Instituto de Fisica da Universidade Federal do Rio Grande do Sul, BR-91500-970 Porto Alegre, Brazil \\ ${ }^{3}$ Helmholtz-Zentrum Berlin für Materialien und Energie GmbH, Institute G-12 Hahn-Meitner Platz, 1, DE-14109 Berlin, Germany
}

(Received 9 January 2013; published 1 April 2013)

\begin{abstract}
The energy loss of an ion passing along a major crystal axis before and/or after undergoing a large-angle collision is strongly enhanced, because its path is close to a lattice-site atom located at the crystal axis; this is the so-called skimming effect. One may find this effect in high-resolution medium-energy ion scattering (MEIS) spectra from submonolayers of single crystals. In the present study, the MEIS spectra were observed for mediumenergy $\mathrm{He}^{+}$ions backscattered from $\mathrm{KI}(001)$ and $\mathrm{RbI}(001)$ crystals at various scattering geometries. The observed surface peaks were decomposed into scattering components from top-, second-, and third-layer atoms considering the hitting probabilities, which were derived from Monte Carlo simulations of ion trajectories, taking account of the enhanced and correlated thermal vibrations. The results obtained here demonstrate that the energy loss of the ions which move skimming trajectories is expressed reasonably well by the impact-parameter-dependent energy loss calculated using the nonperturbative coupled-channel method.
\end{abstract}

DOI: 10.1103/PhysRevA.87.042901

PACS number(s): 34.50.Bw, 68.49.Sf, 79.20.Rf, 61.85.+p

\section{INTRODUCTION}

In ion scattering spectrometry, the energy loss of ions allows for depth-profiling constituent elements of thin films. The energy loss or stopping power values have been compiled in a database semiempirically by Ziegler and co-workers [1-3]. These data, however, can be applied only to polycrystalline and amorphous targets and also to random penetration in crystalline solids. On the other hand, the ions channeling along a crystal axis or plane undergo a smaller mean energy loss than that for the ions passing along a random direction, because the channeled ions penetrate through a region of a low electron density [4-6]. In contrast, the ions incident along a crystal axis and scattered to a large angle or scattered to a blocking direction undergo a large energy loss because they penetrate electron shells close to target nuclei. Recently, Hentz et al. [7] reported trajectory-dependent energy losses of medium-energy protons incident on $\mathrm{Cu}(111)$ crystals. They performed Monte Carlo simulations of ion trajectories as well as coupled-channel calculations of impact-parameterdependent energy loss and demonstrated that the energy spectrum observed for $100-\mathrm{keV} \mathrm{H}^{+}$ions is reproduced well by the simulations.

In the present study, we measured the energy spectra for 70and $80-\mathrm{keV} \mathrm{He}^{+}$ions incident on $\mathrm{KI}(001)$ and $\mathrm{RbI}(001)$ for various scattering geometries. The low Debye temperatures of $130 \mathrm{~K}$ for $\mathrm{KI}$ and $115 \mathrm{~K}$ for $\mathrm{RbI}$ are quite convenient to see clearly the scattering component from the second-layer atoms. The spectra were analyzed in a layer-by-layer fashion due to an excellent energy resolution $\left(\triangle E / E=1.0 \times 10^{-3}\right.$ : full width at half maximum) of a toroidal electrostatic analyzer (ESA) [8]. In order to decompose uniquely an observed surface peak into each scattering component from the top-, second-, and third-layer atoms, we performed Monte Carlo

\footnotetext{
*Author to whom correspondence should be addressed: ykido@se.ritsumei.ac.jp
}

simulations of He ion trajectories considering enhanced and correlated thermal vibrations. The difference between the emerging energies $\left(\Delta E_{1-2}\right)$ for the ions scattered from the topand second-layer atoms corresponds to the ion path between first and second layer only. As both lines are observed for the same backscattering atom in the same backscattering direction, the kinematic (quasielastic) energy reduction as well as the enhanced inner-shell excitation is identical for both backscattering events. The electronic surface position (effective jellium edge) is always in front of the surface defined by the target nuclear positions and this distance is similar to the interaction range that determines electronic excitations during the collision with a backscattering atom in the second layer. Thus, for the ion path corresponding to $\Delta E_{1-2}$, we need to consider only the incident and emerging angles of the ions and the nuclear interlayer distance. The resulting energy-loss difference corresponds to two processes: (i) local energy loss incurred during passing close to a lattice-site atom (skimming effect), and (ii) average energy loss proportional to the path length. The latter corresponds to the random stopping power. The results obtained experimentally are compared with theoretical predictions based on the coupled-channel method $[9,10]$ that does not rely on a perturbative treatment of the interaction dynamics.

\section{EXPERIMENT}

Medium-energy $\mathrm{He}^{+}$ions were provided by a duo-plasma ion source coupled with a direct current power supply with a voltage ripple better than $5 \times 10^{-4}$. After acceleration, a switching magnet selected the ion species and energy. The ion beam was then collimated to a size of $0.18 \times 2.0 \mathrm{~mm}^{2}$ in the horizontal and vertical directions, respectively, before impinging on a target surface. The energies of scattered $\mathrm{He}^{+}$ ions were selectively analyzed by the toroidal electrostatic analyzer with a central deflection radius of $150 \mathrm{~mm}$. The entrance slit size was $0.2 \times 4.0 \mathrm{~mm}^{2}$ in the horizontal and 
vertical directions, respectively, and the narrow slit opening in the horizontal plane is responsible for the energy selection. The deflected $\mathrm{He}^{+}$ions arrived at a three-stage microchannel plate combined with a position-sensitive detector $(20 \mathrm{~mm}$ in diameter) of a semiconductor ( $\mathrm{Si}$ type) named PIAS (Hamamatsu Photonics) whose position resolution was $40 \mu \mathrm{m}$. The large interelectrode distance of $16 \mathrm{~mm}$ covers a wide energy range ( $\sim 8 \%$ of the pass energy) for deflected $\mathrm{He}^{+}$ ions at a constant voltage applied on the toroidal electrodes. The condition mentioned above gives rise to the excellent energy resolution of $1.0 \times 10^{-3}$ without degrading the statistics.

The $\mathrm{KI}(001)$ and $\mathrm{RbI}(001)$ crystals take a NaCl-type structure with lattice constants of 7.066 and $7.342 \AA$, respectively. A crystal substrate with a size of $14 \times 14 \times 1 \mathrm{~mm}^{3}$ was obtained by cleaving the bulk crystal in the atmosphere and was then immediately introduced into a scattering chamber evacuated to an ultrahigh vacuum (UHV: $\sim 2 \times 10^{-10}$ Torr). Before the MEIS measurement, infrared radiation heated the substrate to $\sim 150^{\circ} \mathrm{C}$ for $5 \mathrm{~min}$ to eliminate contamination [11]. A clean surface without $\mathrm{C}$ and $\mathrm{O}$ adsorption was confirmed by Auger electron spectroscopy.

The sample surface was covered with $\mathrm{Al}$ foils except for an ion-irradiation area to be charge-up free and positively biased at $90 \mathrm{~V}$ to suppress secondary electron emission. In spite of that, in many cases, the slope of the front edges of observed MEIS spectra was smeared out significantly in comparison with the expected system energy resolution, probably due to a slight charge-up. In order to avoid radiation damage, we shifted the irradiated area by $\sim 0.2 \mathrm{~mm}$ in the horizontal direction after accumulating a beam current of $1 \mu \mathrm{C}$.

\section{MONTE CARLO SIMULATIONS OF ION TRAJECTORIES}

In order to deduce accurately the emerging-energy difference between the scattering components from the top- and second-layer atoms, the observed surface peak should be decomposed uniquely. For this purpose, it is essential to see the hitting probability for the atoms of each layer as well as the line shape for each scattering component. Concerning the latter, we employed the exponentially modified Gaussian (EMG) line shape [12,13] and the Lindhard-Scharff formula [14] to calculate the energy straggling, whose reliabilities were confirmed experimentally in advance [13,15-17]. The hitting probability for the atoms of each layer is calculated by Monte Carlo (MC) simulations of ion trajectories, which are explained in detail below.

Figure 1 illustrates the scattering geometries taken in the present MEIS spectrum observation. The surfaces are slightly relaxed (KI: $-1.6 \%, \mathrm{RbI}:-1.1 \%)$ and rumpled (KI: 1.8\%, RbI: $2.2 \%$ ) $[11,18,19]$ but such reconstructions were neglected in the present MC simulations, which give the hitting probabilities (close encounter probabilities: $P_{\mathrm{CL}}$ ) for the second- and third-layer atoms normalized by that for the top-layer atoms. In this Monte Carlo simulation, we assumed root-mean-square (rms) one-dimensional (1D) bulk thermal vibration amplitudes $\left(u_{\text {bulk }}\right)$ of 0.20 and $0.205 \AA$, respectively, for $\mathrm{I}$ and $\mathrm{Rb}$ of $\mathrm{RbI}(001)$ and 0.190 and $0.195 \AA$, respectively, for I and $\mathrm{K}$ of $\mathrm{KI}(001)$ determined experimentally $[11,18,19]$. In addition, also assumed are the correlations calculated from molecular dynamics using the Catlow potentials [20] and the thermal vibration amplitude of the top-layer atoms in the surface normal direction enhanced by $\sqrt{2}$ compared with
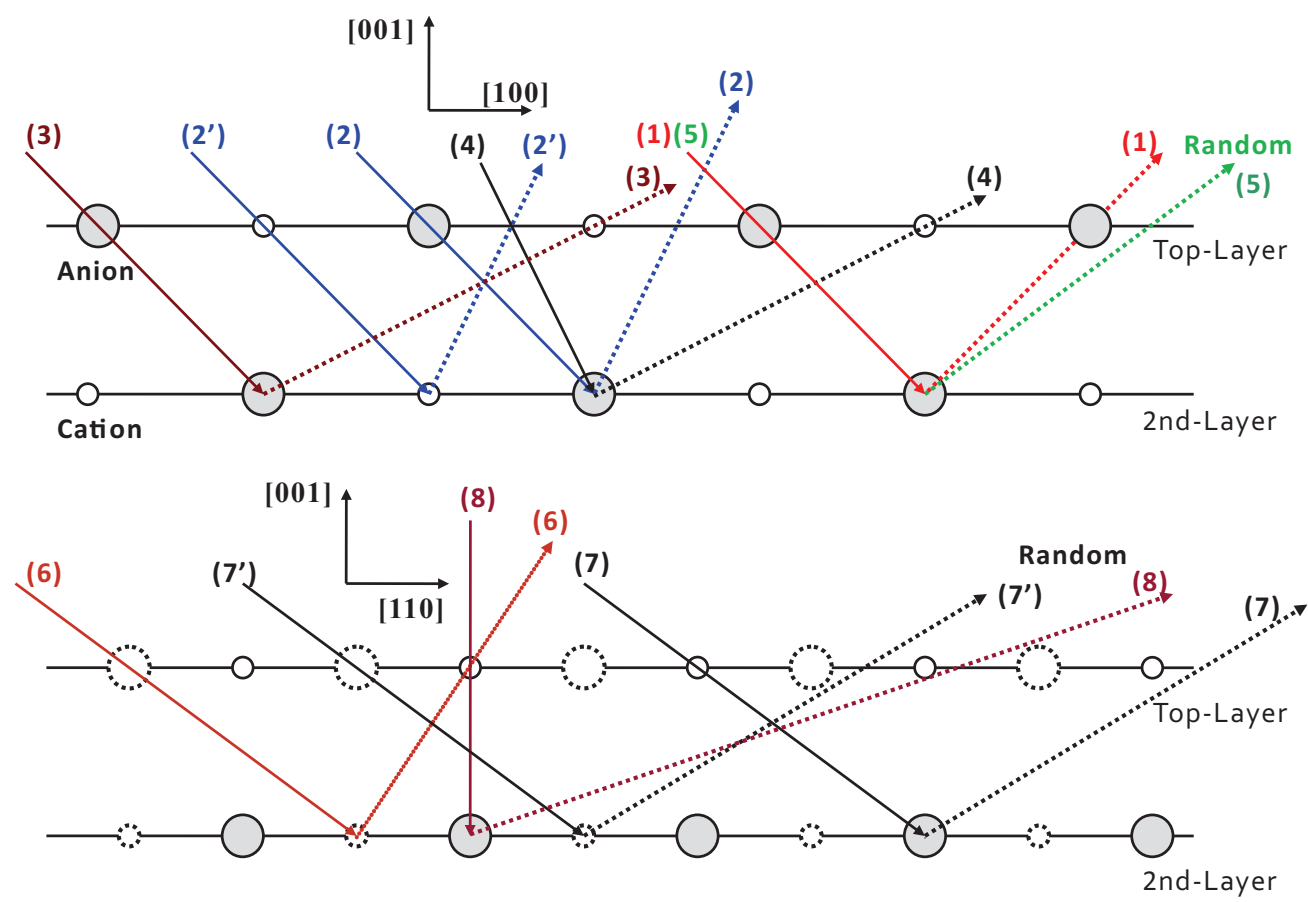

FIG. 1. (Color online) Scattering geometries taken in the MEIS measurements. Small and large circles denote cation and anion, respectively.

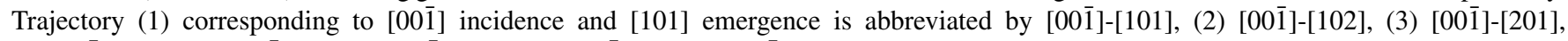

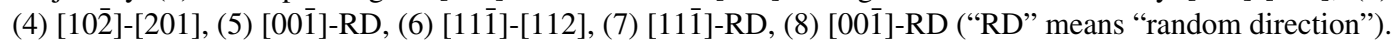


the $u_{\text {bulk }}$. The normalized hitting probability for the $n$ th-layer atoms located in a crystal string ( $z$ axis) is calculated assuming a single-row approximation [21] by

$$
\begin{aligned}
P_{\mathrm{CL}}(n)= & \int \cdots \int \varphi\left(\vec{r}_{1}, \vec{r}_{2}, \vec{r}_{3}, \ldots, \vec{r}_{n-1}, \vec{r}_{0}+\vec{\Delta}_{n}\right) \\
& \times d^{2} \vec{r}_{1} d^{2} \vec{r}_{2} \ldots d^{2} \vec{r}_{n-1} d^{2} \vec{r}_{0} \\
= & \int \cdots \int \varphi\left(\xi_{1}, \xi_{2}, \xi_{3}, \ldots, \xi_{2 n-1}, \xi_{2 n}\right) \\
& \times d \xi_{1} d \xi_{2} d \xi_{3} \ldots d \xi_{2 n-1} d \xi_{2 n} \\
= & Q \int \ldots \int \frac{\varphi\left(\xi_{1}, \xi_{2}, \ldots, \xi_{2 n}\right)}{q\left(\xi_{1}, \xi_{2}, \ldots, \xi_{2(n-1)}\right)} \\
& \times \frac{q\left(\xi_{1}, \xi_{2}, \ldots, \xi_{2(n-1)}\right)}{Q} d \xi_{1} d \xi_{2}, \ldots, d \xi_{2 n} \\
\cong & \frac{Q}{N} \sum_{j=1}^{N} \frac{\varphi\left(\xi_{1}^{(j)}, \xi_{2}^{(j)}, \ldots, \xi_{2 n}^{(j)}\right)}{q\left(\xi_{1}^{(j)}, \xi_{2}^{(j)}, \ldots, \xi_{2(n-1)}^{(j)}\right)} .
\end{aligned}
$$

$\xi_{1}=\frac{x_{1}}{\sigma_{x}^{(1)}}, \xi_{2}=\frac{y_{1}}{\sigma_{y}^{(1)}}, \ldots, \xi_{2 n-3}=\frac{x_{n-1}}{\sigma_{x}^{(n-1)}}, \xi_{2 n-2}=\frac{y_{n-1}}{\sigma_{y}^{(n-1)}}, \xi_{2 n-1}=$ $\frac{X_{n}}{\sigma_{x}^{(n)}}, \xi_{2 n}=\frac{Y_{n}}{\sigma_{y}^{(n)}}$, where $\sigma_{x}^{(n)}$ is a rms 1D thermal vibration amplitude of the $n$ th-layer atoms in the $x$ direction and $\left(\vec{r}_{1}, \vec{r}_{2}, \ldots, \vec{r}_{n-1}\right)$ is the correlated $2(n-1)$-dimensional position vector. Here, the $2 n$-variate normal probability distribution function is expressed by $2 n$-dimensional correlation matrix $S$.

$$
\begin{aligned}
\varphi\left(\xi_{1}, \xi_{2}, \ldots, \xi_{2 n}\right) \equiv & \frac{1}{\sqrt{(2 \pi)^{2 n}\left|S_{2 n}\right|}} \\
& \times \exp \left[-\frac{1}{2} \sum_{i=1}^{2 n} \sum_{k=1}^{2 n}\left(S_{2 n}^{-1}\right)_{i k} \xi_{i} \xi_{k}\right] \\
q\left(\xi_{1}, \xi_{2}, \ldots, \xi_{2(n-1)}\right)= & \frac{1}{A} \frac{1}{\sqrt{(2 \pi)^{2(n-1)}\left|S_{2(n-1)}\right|}} \\
& \times \exp \left[-\frac{1}{2} \sum_{i=1}^{2(n-1)} \sum_{k=1}^{2(n-1)}\left(S_{2(n-1)}^{-1}\right)_{i k} \xi_{i} \xi_{k}\right], \\
Q \equiv & \int \ldots \int q\left(\xi_{1}, \xi_{2}, \ldots, \xi_{2(n-1)}\right) d \xi_{1} \\
& \times d \xi_{2} \cdots d \xi_{2(n-1)}=1,
\end{aligned}
$$

where $\vec{R}_{0}$ is a random starting position, $A$ an incident area, $\vec{R}_{n}:\left(X_{n}, Y_{n}\right) \equiv \vec{R}_{0}+\vec{\Delta}_{n}$ the incident position in the $n$th layer, $N$ the number of incident ions, and $S$ is the correlation matrix given by $S_{i j}=\left\langle\xi_{i} \cdot \xi_{j}\right\rangle$ ( \langle\rangle means a time average). The $\xi \equiv\left(\xi_{1}, \xi_{2}, \ldots, \xi_{2(n-1)}\right)$ is a correlated $2(n-1)$-variate normal distribution expressed by $\xi=T^{-1} \eta$, where $\eta \equiv$ $\left(\eta_{1}, \eta_{2}, \ldots, \eta_{2(n-1)}\right)$ is the normalized position vector generated by assembling the normal distribution of the $2(n-1)$-univariate independent random variables, and the transformation matrix $T$ is given by $\tilde{T} T=S^{-1}$ [22]. A nuclear encounter would have taken place if the atom residing in the $n$th layer were located exactly at $\vec{r}_{n}=\vec{R}_{n}\left(=\vec{R}_{0}+\vec{\Delta}_{n}\right)$. Note that only the coordinates in the $(x, y)$ planes are relevant to the problem. We also assumed that the motion in the $x$ direction is independent of that in the $y$ direction. The basic treatment to calculate the hitting probabilities considering the correlations is given in the literature [21]. Thus the surface peak observed was deconvoluted uniquely into each scattering component from

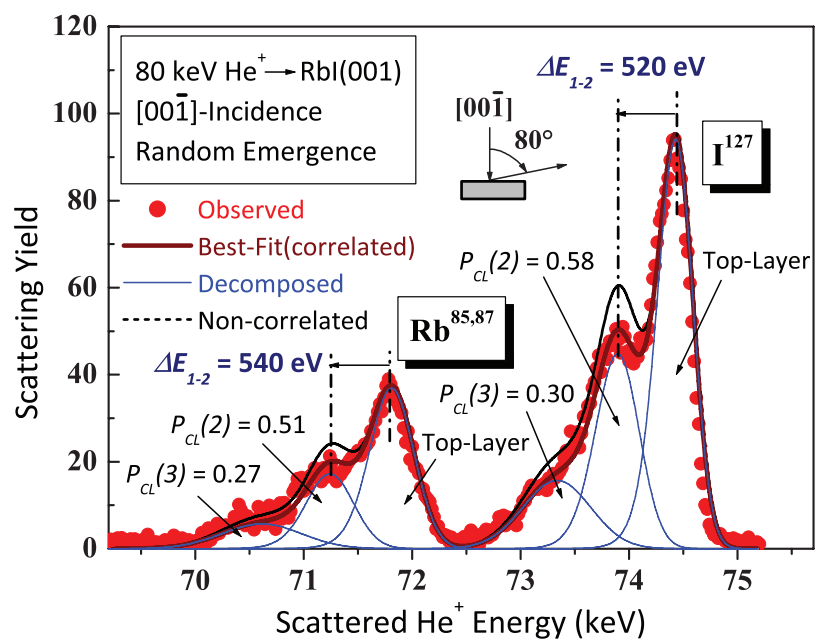

FIG. 2. (Color online) MEIS spectrum (filled circles) observed for $80-\mathrm{keV} \mathrm{He}^{+}$ions incident along the [001] axis of $\mathrm{RbI}(001)$ and scattered to $100^{\circ}$, about $2^{\circ}$ off from [100] azimuth (random emergence). Thick (brown) and thin (blue) solid curves denote best-fitted spectrum and scattering components from top-, secondand third-layer atoms, respectively. Best-fitted spectrum was obtained by assuming energy difference $\Delta E_{1-2}=520 \mathrm{eV}$ for I and $540 \mathrm{eV}$ for $\mathrm{Rb}$ and hitting probabilities of 0.58 and 0.30 for the secondand third-layer I atoms and 0.51 and 0.27 for $\mathrm{Rb}$ atoms in [001] crystal axis, which correspond to correlations of +0.35 and +0.10 , respectively, between first and second nearest-neighbor atoms.

the top-, second-, and third-layer atoms, considering the hitting probabilities (assumed to be highly accurate) calculated from the MC simulations of $\mathrm{He}$ ion trajectories described above.

\section{RESULTS AND DISCUSSION}

Figure 2 shows a typical MEIS spectrum (filled circles) observed for $80-\mathrm{keV} \mathrm{He}^{+}$ions incident along the [001] axis of $\mathrm{RbI}(001)$ and scattered to $100^{\circ}$ (random direction: $2^{\circ}$ off from [100] azimuth; see Fig. 1: trajectory (8)). The surface peaks are decomposed into three scattering components from the top-, second-, and third-layer I and Rb atoms (solid curves). Note that the shadowing effect was weakened owing to large root-mean-square thermal vibration amplitudes expected from the low Debye temperature of $115 \mathrm{~K}$. Here, we employed the EMG function as the line shape $[12,13]$. The best fit was obtained by assuming the energy differences $\left(\Delta E_{1-2}\right)$ of $520 \pm 20$ and $540 \pm 30 \mathrm{eV}$ between the scattering components from the top- and second-layer I and $\mathrm{Rb}$ atoms, respectively. In order to fit the leading edge, it was also assumed that the energy straggling took a value of $0.35 \Omega_{B}^{2}\left(\Omega_{B}\right.$ : Bohr straggling) [14,15], which is significantly larger than that given by the Lindhard-Scharff formula. This is probably due to the slight charge-up, as mentioned before. The hitting probabilities $\left(P_{\mathrm{CL}}\right)$ deduced here for the second- and third-layer I and $\mathrm{Rb}$ atoms indicate significant correlations of +0.35 and +0.10 , respectively, between the first and second nearest-neighbor atoms in the [001] string for the motion perpendicular to this string. Here, the positive correlation represents an attractive motion caused by acoustic phonon modes, which lowers the close encounter probability because of a stronger shadowing 


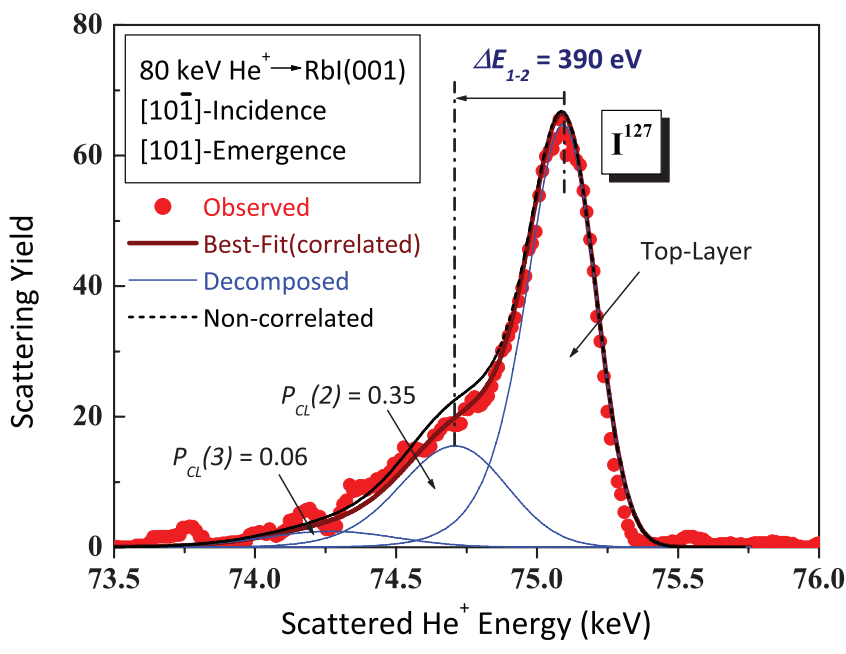

FIG. 3. (Color online) MEIS spectrum (filled circles) observed for $80-\mathrm{keV} \mathrm{He}^{+}$ions incident along [101] axis of $\mathrm{RbI}(001)$ and scattered from I to [101] direction. Notation is the same as that in Fig. 2. Best-fitted spectrum was obtained by assuming energy difference $\Delta E_{1-2}=390 \mathrm{eV}$ for scattering component from I and hitting probabilities of 0.35 and 0.06 , respectively, for the secondand third-layer I atoms in the [101] string, which correspond to a correlation of +0.14 between first nearest-neighbor I atoms.

effect. Note that acoustic phonon modes, rather than optical modes, are generally dominant because the number of created phonons is proportional to the density of state multiplied by the Planck distribution function. Figure 3 shows the MEIS spectrum observed for $80-\mathrm{keV} \mathrm{He}^{+}$ions incident along the [10 $\overline{1}$ ] axis of $\operatorname{RbI}(001)$ and backscattered to the [101] direction [see Fig. 1: trajectory (1)]. A best-fitted MEIS spectrum was obtained assuming the energy difference of $\Delta E_{1-2}=390 \pm 10 \mathrm{eV}$ and a significant correlation of +0.15 between the first nearest-neighbor I atoms in the [101] string. The smaller correlation for the [101] string compared with that for the [001] string is attributed to the larger interatomic distance by $\sqrt{2}$. The correlations estimated here are consistent with those calculated from molecular dynamics simulations reported previously $[11,18,19]$. This evidences the validity of the present deconvolution procedure.

From the energy differences $\left(\Delta E_{1-2}\right)$ derived here for 80-keV $\mathrm{He}^{+}$ions scattered from $\mathrm{RbI}$ for various kinds of scattering geometries, as indicated in Table I, we can deduce the local atomic energy loss (skimming effect) and random energy loss $\left(S_{e}^{\mathrm{RbI}}: \mathrm{eV} / \AA \AA\right)$ proportional to the path length. For simplicity, as the first approximation, it is assumed here that the local energy loss is subjected to the He ions during passage through a major crystalline axis, while the ions penetrating along random directions undergo a random energy loss. Later we constrain the region of the local energy loss within a Wigner-Seitz cell to match the coupled-channel calculations. The energy difference $\left(\Delta E_{1-2}\right)$ observed for the [101] incidence and backscattered by I atoms to the [101] direction, the local energy loss subjected by skimming through the top-layer I atoms $\Delta E(\mathrm{I})$ is determined to be $195 \pm 10 \mathrm{eV}$ (just half of the energy difference $\Delta E_{1-2}=390 \mathrm{eV}$ ). This local energy loss value would correspond to $(195 \mathrm{eV}) /(5.19 \AA)=$ $37.6 \mathrm{eV} / \AA$, which is much larger than the Ziegler's random stopping power of $12 \mathrm{eV} / \AA$ for $80-\mathrm{keV} \mathrm{He}^{+}$ions passing through RbI. The local energy loss subjected by passing close to a $\mathrm{Rb}$ atom $\Delta E(\mathrm{Rb})$ is then derived to be $190 \pm 15 \mathrm{eV}$ and the random energy loss $\left(S_{e}^{\mathrm{RbI}}\right)$ of $15.8 \mathrm{eV} / \AA$ is obtained from the energy difference $\left(\Delta E_{1-2}\right)$ observed for the [10i]] incidence and backscattered from $\mathrm{Rb}$ and I atoms to the [102] direction [see Table I and also Fig. 1: trajectory (2)]. The random energy loss values are also deduced to be 15.6 and $16.3 \mathrm{eV} / \AA$, respectively, from the energy differences $\left(\Delta E_{1-2}\right)$ observed for the [001] incidence and scattered to $100^{\circ}$ using the above local energy loss values [see Fig. 1: trajectory (8)]. Combined with the energy differences derived for the two scattering geometries, $[00 \overline{1}]$ incidence and scattered to $100^{\circ}$ [trajectory (8)] and [11 $\overline{1}$ ] incidence and scattered to the [112]

TABLE I. Energy difference $\left(\Delta E_{1-2}\right)$ between the scattering components from top- and second-layer atoms measured for various scattering geometries. Incident $\mathrm{He}^{+}$ions collide with (atom) leading to backscattering and incident or scattered He ions skim through $\langle$atom $\rangle$. The number indicated in the "Scattering geometry" column corresponds to that in Fig. 1. "RD" means "random direction." Local energy losses are calculated assuming $S_{e}^{\mathrm{KI}}=14 \mathrm{eV} / \AA$ and $S_{e}^{\mathrm{RbI}}=16 \mathrm{eV} / \AA$.

\begin{tabular}{|c|c|c|c|c|c|c|c|}
\hline Target & $\begin{array}{l}\text { Energy } \\
(\mathrm{keV})\end{array}$ & $\begin{array}{l}\text { Scattering geometry } \\
\quad \text { (see Fig. 1) }\end{array}$ & $\begin{array}{c}\text { Colliding } \\
\text { with (atom) } \\
\text { and skimming } \\
\text { through }\langle\text { atom }\rangle\end{array}$ & $\begin{array}{c}\text { Energy } \\
\text { difference } \\
\Delta E_{1-2}(\mathrm{eV})\end{array}$ & $\begin{array}{l}\text { Path length } \\
\text { incoming- } \\
\text { outgoing }(\AA)\end{array}$ & $\begin{array}{c}\text { Path } \\
\text { length } \\
\text { for random } \\
\text { energy loss }(\AA)\end{array}$ & $\begin{array}{c}\text { Local energy } \\
\text { loss }(\mathrm{eV})\end{array}$ \\
\hline KI & 70 & (5) & $\langle\mathrm{I}\rangle-(\mathrm{I})$ & $260 \pm 10$ & $5.0-6.16(\mathrm{RD})$ & 6.78 & $165.08\langle\mathrm{I}\rangle$ \\
\hline KI & 70 & (1) & $\langle\mathrm{I}\rangle-(\mathrm{I})-\langle\mathrm{I}\rangle$ & $350 \pm 10$ & $5.0-5.0$ & 1.23 & $166.39\langle\mathrm{I}\rangle$ \\
\hline KI & 70 & $\left(7^{\prime}\right)$ & $\langle\mathrm{I}\rangle-(\mathrm{K})$ & $305 \pm 15$ & $6.12-8.28(\mathrm{RD})$ & 10.0 & $165.0\langle\mathrm{I}\rangle$ \\
\hline KI & 70 & (7) & $\langle\mathrm{K}\rangle-(\mathrm{I})$ & $260 \pm 10$ & $6.12-8.28(\mathrm{RD})$ & 10.0 & $120.0\langle\mathrm{~K}\rangle$ \\
\hline $\mathrm{KI}$ & 70 & (3) & $\langle\mathrm{I}\rangle-(\mathrm{I})-\langle\mathrm{K}\rangle$ & $315 \pm 10$ & $5.0-7.90$ & 4.13 & $257.2\langle\mathrm{~K}+1\rangle$ \\
\hline $\mathrm{RbI}$ & 80 & (8) & $\langle\mathrm{Rb}\rangle-(\mathrm{I})$ & $520 \pm 20$ & 3.67-21.14(RD) & 20.26 & $195.84\langle\mathrm{Rb}\rangle$ \\
\hline $\mathrm{RbI}$ & 80 & $\left(8^{\prime}\right)$ & $\langle\mathrm{I}\rangle-(\mathrm{Rb})$ & $540 \pm 30$ & $3.67-21.14(\mathrm{RD})$ & 20.26 & $215.84\langle\mathrm{I}\rangle$ \\
\hline $\mathrm{RbI}$ & 80 & (6) & $\langle\mathrm{I}\rangle-(\mathrm{Rb})$ & $265 \pm 15$ & $6.36-4.50(\mathrm{RD})$ & 6.31 & $164.04\langle\mathrm{I}\rangle$ \\
\hline $\mathrm{RbI}$ & 80 & (4) & $(\mathrm{I})-\langle\mathrm{Rb}\rangle$ & $270 \pm 10$ & $4.10(\mathrm{RD})-8.21$ & 7.76 & $145.84\langle\mathrm{Rb}\rangle$ \\
\hline $\mathrm{RbI}$ & 80 & (1) & $\langle\mathrm{I}\rangle-(\mathrm{I})-\langle\mathrm{I}\rangle$ & $390 \pm 10$ & $5.19-5.19$ & 1.27 & $184.84\langle\mathrm{I}\rangle$ \\
\hline $\mathrm{RbI}$ & 80 & (2) & $\langle\mathrm{I}\rangle-(\mathrm{I})$ & $260 \pm 10$ & 5.19-4.10(RD) & 4.74 & $184.16\langle\mathrm{I}\rangle$ \\
\hline $\mathrm{RbI}$ & 80 & $\left(2^{\prime}\right)$ & $\langle\mathrm{Rb}\rangle-(\mathrm{Rb})$ & $255 \pm 15$ & 5.19-4.10(RD) & 4.74 & $179.16\langle\mathrm{Rb}\rangle$ \\
\hline
\end{tabular}


direction [trajectory (6)], we obtain the random energy loss of $16.5 \mathrm{eV} / \AA$. The local energy loss for $\mathrm{Rb}$ is also derived to be $\Delta E(\mathrm{Rb})=206 \pm 4 \mathrm{eV}$ from the $\Delta E_{1-2}$ observed for the [102]] incidence and scattered to the [201] direction [see Fig. 1: trajectory (4)]. However, in this scattering geometry the path length along the [201] axis (skimming path) is too long $(8.21 \AA)$. This probably gives an apparently larger local energy loss value. Therefore, it is reasonable to constrain the region of the atomic (local) energy loss within a critical volume, for example, a Wigner-Seitz cell. Indeed, this treatment matches the coupled-channel approach in which the excitation and ionization probabilities are calculated along the incident ion path. Here, we assume that the volume of the Wigner-Seitz cell is equal to the volume of a sphere, $4 \pi R^{3} / 3$, and thus we obtain the radius of $R=2.277$ for $\mathrm{RbI}$ and $2.192 \AA$ for KI. The path length for random energy loss is corrected by subtracting $2 R \times$ (number of skimming atoms) from the total path length, as indicated in Table I. In the case of the $[10 \overline{1}]$ incidence and backscattered from I to the [101] direction [see Fig. 1: trajectory (1)], we neglected the random energy loss. This should lower slightly the local energy loss determined previously for He ions skimming through an I atom, while slightly increase the random energy loss. Note that the interatomic distance in the [101] string is slightly longer than the diameter of the Wigner-Seitz sphere and thus the correction is small enough. At present we adopt the averaged random energy loss value of $16 \mathrm{eV} / \AA$ in spite of some uncertainty, roughly estimated to be $\pm 1 \mathrm{eV} / \AA$. The corrected local energy loss values for I and $\mathrm{Rb}$ are shown in Table I. Thus we obtain the average local energy losses of $\Delta E(\mathrm{I})=187 \pm 20$ and $\Delta E(\mathrm{Rb})=174 \pm 20 \mathrm{eV}$ for $80-\mathrm{keV}$ $\mathrm{He}^{+}$ions skimming through an I and $\mathrm{Rb}$ atom of $\mathrm{RbI}(001)$, respectively.

A similar analysis was also carried out for $70-\mathrm{keV} \mathrm{He}^{+}$ ions incident on $\mathrm{KI}(001)$. Figures 4 and 5 show the MEIS spectra observed for $70-\mathrm{keV} \mathrm{He}^{+}$ions incident along the [101] axis and scattered from I atoms to the [101] direction, and incident along the [11 $\overline{1}]$ axis and scattered from $\mathrm{K}$ atoms to a random direction [see Fig. 1: trajectory $\left(7^{\prime}\right)$ ], respectively. For the $[10 \overline{1}]$ incidence, the observed MEIS spectrum was best fitted assuming the energy difference $\left(\Delta E_{1-2}\right)$ of $350 \pm 10 \mathrm{eV}$ and the hitting probabilities of 0.35 and 0.03 for the secondand third-layer I atoms, indicating no correlation, while for the [11 1$]$ incidence the $\Delta E_{1-2}$ value is $305 \pm 15 \mathrm{eV}$ and the hitting probabilities are 0.519 and 0.238 for the second- and third-layer $\mathrm{K}$ atoms, corresponding to a correlation of +0.10 between the neighboring $\mathrm{K}$ and $\mathrm{I}$ atoms in the [111] string. We also found a significant correlation of +0.20 between

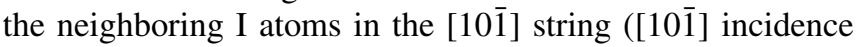
and random emergence, not shown here). That no correlation was found for the [10 $\overline{1}]$-[101] double-alignment geometry is possibly due to a smaller correlation in the [101] string rather than that in the [001] string or to a slight misalignment. The correlations derived here are consistent with those calculated from molecular dynamics analysis, indicating the validity of the convolution procedure $[11,18,19]$ also for the $\mathrm{KI}(001)$ target. From the energy difference $\Delta E_{1-2}=350 \pm 10 \mathrm{eV}$ observed for the [10 $\overline{1}]$ incidence and [101] emergence, the local energy loss for $70-\mathrm{keV} \mathrm{He}^{+}$ions skimming through an I atom is determined to be $175 \pm 10 \mathrm{eV}$, which is significantly

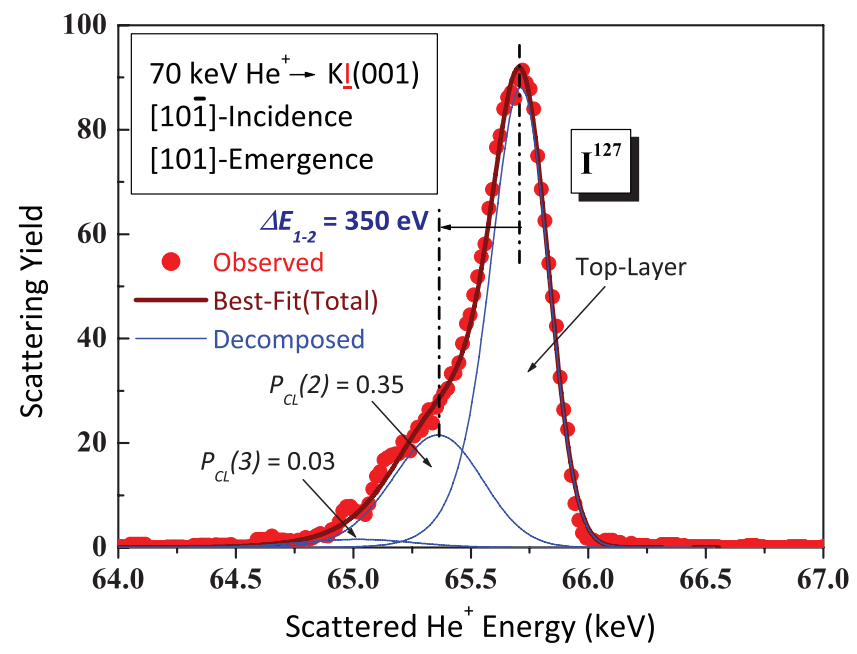

FIG. 4. (Color online) MEIS spectrum (filled circles) observed for $70-\mathrm{keV} \mathrm{He}^{+}$ions incident along the [101] axis of $\mathrm{KI}(001)$ and scattered from I atoms to the [101] direction. Thick (brown) and thin (blue) solid curves denote best-fitted spectrum and scattering components from top-, second-, and third-layer atoms, respectively. The best-fitted spectrum was obtained by assuming energy difference $\Delta E_{1-2}=350 \mathrm{eV}$ for scattering component from I and hitting probabilities of 0.35 and 0.03 , respectively, for the second- and third-layer I atoms in the [101] string, which correspond to no correlations.

smaller than that $(195 \pm 10 \mathrm{eV})$ for $80-\mathrm{keV} \mathrm{He}^{+}$ions. This local energy loss of $175 \mathrm{eV}$ corresponds to $35 \mathrm{eV} / \AA$, which is much larger than the Ziegler's random energy loss of $10.5 \mathrm{eV} / \AA$. Then we obtained the random energy loss value, $S_{e}^{\mathrm{KI}}$ of $13.8 \mathrm{eV} / \AA$, from the energy difference $\left(\Delta E_{1-2}\right)$

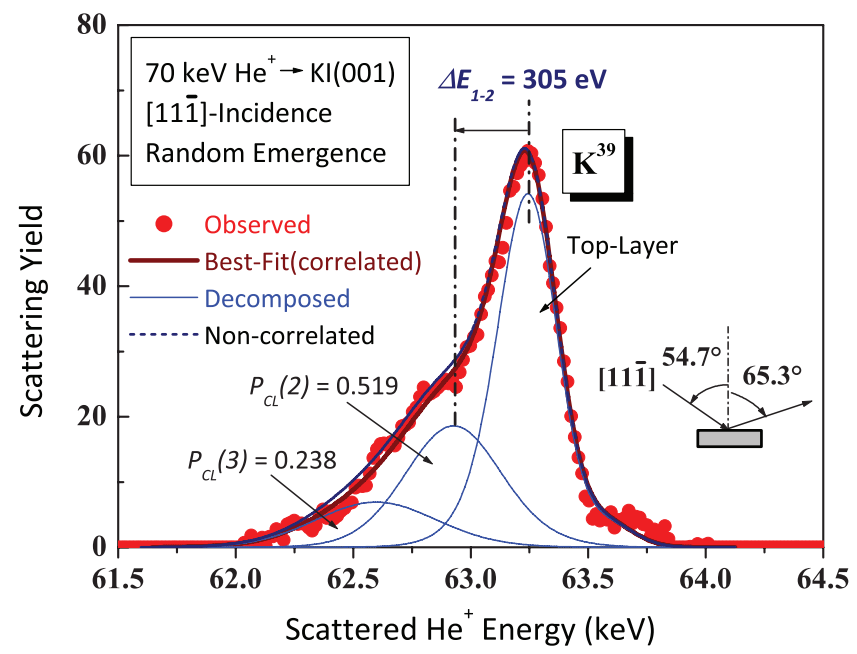

FIG. 5. (Color online) MEIS spectrum observed with 70-keV $\mathrm{He}^{+}$ions incident along the [11i] ] axis of $\mathrm{KI}(001)$ and scattered from $\mathrm{K}$ to $65.3^{\circ}$ with respect to surface normal (random direction). Notation is the same as that in Fig. 4. Best-fitted spectrum was obtained by assuming energy difference $\Delta E_{1-2}=305 \mathrm{eV}$ for scattering component from $\mathrm{K}$ and hitting probabilities of 0.52 and 0.24 , respectively, for the second- and third-layer $\mathrm{K}$ atoms in the [111] string, which correspond to a correlation of +0.10 between the neighboring $\mathrm{K}$ and $\mathrm{I}$ atoms. 
observed for the [101]] incidence and random emergence (see Table I). As mentioned before for $\mathrm{RbI}(001)$, we must correct the path length for random energy loss. Here, we adopt the random energy loss value of $14 \mathrm{eV} / \AA$, slightly larger than the above value. The corrected path lengths and the local energy loss values estimated assuming the random energy loss of $14 \mathrm{eV} / \AA$ are indicated in Table I. The local energy loss value for an I atom averaged for three different geometries is $165.5 \mathrm{eV}$ with a slight dispersion of $\pm 1 \mathrm{eV}$. The energy difference $\left(\Delta E_{1-2}\right)$ observed for the [11 $\left.\overline{1}\right]$ incidence and scattered to $65.26^{\circ}$ with respect to surface normal (random) gives the local energy loss of $\Delta E(\mathrm{~K})=120 \mathrm{eV}$ [see Fig. 1: trajectory (7)]. The local energy loss of $257.2 \mathrm{eV}$ for He ions skimming through a $\mathrm{K}$ and I atom estimated from the data observed for the [10 $\overline{1}$ ] incidence and [201] emergence [see Fig. 1: trajectory (3)] is smaller by about $10 \%$ than that expected from the other scattering geometries $(120+165.5=$ $285.5 \mathrm{eV})$, indicating roughly the uncertainty of $\pm(10-20) \mathrm{eV}$.

We calculated the impact-parameter $(b)$-dependent energy loss by the coupled-channel method [9]. Shortly, the timedependent Schrödinger equation was solved for one active target electron in the framework of the independent particle model. The projectile-electron interaction was described by a single-zeta potential for $\mathrm{He}^{+}$projectiles. Excitation and ionization probabilities were numerically calculated shellby-shell allowing the contributions of transitions for about 500 discrete and continuum (wave packet) states with orbital quantum numbers up to $l=8$ and energies up to $2 m_{e} v^{2}$ ( $m_{e}$ : electron mass and $v$ : ion velocity). The average energy transfers for $70-$ and $80-\mathrm{keV}$ He ions to each electron from different subshells of $\mathrm{I}, \mathrm{K}$, and $\mathrm{Rb}$ atoms are indicated in Table II. Here, we assumed only near-central collisions ( $b$ close to 0 ) and a frozen ionic state of $\mathrm{He}^{+}$.

The local energy losses determined from the MEIS observation are compared with the theoretical values calculated from the coupled-channel method in Table III. The local energy losses for $80-\mathrm{keV} \mathrm{He}^{+}$ions skimming through a $\mathrm{Rb}$ and an I atom determined from the present MEIS spectrum analysis agree well with the theoretical predictions. Surprisingly, the local energy loss for $70-\mathrm{keV} \mathrm{He}^{+}$ions passing close to a ${ }^{19} \mathrm{~K}$ atom calculated from the coupled-channel method is significantly larger than that for the $\mathrm{He}^{+}$ions penetrating close to an ${ }^{53} \mathrm{I}$ atom despite the smaller number of total electrons. Note, however, that the number of electrons easily removed according to the criterion, $2 m_{e} v^{2}>I_{b}\left(2 m_{e} v^{2}\right.$ is the maximum energy transfer in a free projectile-electron collision and $I_{b}$ is the shell binding energy) is larger for ${ }^{19} \mathrm{~K}$. Thus, the observed local energy loss values are basically consistent with the coupled-channel calculations, although the $\Delta E(\mathrm{~K})$ value derived experimentally is smaller than the calculated one by almost $30 \%$. This deviation may come from the assumed independent particle model that does not consider dynamical screening and increased binding energy in multiple ionizations, which may be more important for the case of slow He projectiles compared to protons. Other uncertainties of the present calculations such as the use of a target-centered basis set (not suitable to describe capture events) and frozen projectile charge state lower than the corresponding equilibrium value may also be responsible for the observed disagreement.
TABLE II. Shell-by-shell energy loss values calculated from the coupled-channel method for $70-\mathrm{keV} \mathrm{He}^{+}$ions' impact on $\mathrm{KI}(001)$ and $80-\mathrm{keV} \mathrm{He}^{+}$ions incident on $\mathrm{RbI}(001)$. The impact parameter is assumed to be 0 (head-on-collision), and electronic shells with binding energies exceeding $600 \mathrm{eV}$ have been excluded. The numbers in parentheses indicate the electron occupancies.

\begin{tabular}{|c|c|c|c|}
\hline \multicolumn{2}{|c|}{$70-\mathrm{keV} \mathrm{He}{ }^{+} \rightarrow{ }^{19} \mathrm{~K}$} & \multicolumn{2}{|c|}{$70-\mathrm{keV} \mathrm{He}^{+} \rightarrow{ }^{53} \mathrm{I}$} \\
\hline Shell & $\begin{array}{l}\text { Energy loss } \\
\text { (eV/electron) }\end{array}$ & Shell & $\begin{array}{l}\text { Energy loss } \\
\text { (eV/electron) }\end{array}$ \\
\hline $2 s(2)$ & 4.0 & $4 s(2)$ & 3.0 \\
\hline $2 p 0(2)$ & 1.6 & $4 p 0(2)$ & 4.2 \\
\hline $2 p 1 g(2)$ & 0.0 & $4 p 1 g(2)$ & 1.7 \\
\hline $2 p 1 u(2)$ & 0.0 & $4 p 1 u(2)$ & 0.9 \\
\hline $3 s(2)$ & 21.9 & $4 d 0(2)$ & 15.6 \\
\hline $3 p 0(2)$ & 30.1 & $4 d 1 g(2)$ & 4.7 \\
\hline $3 p 1 g(2)$ & 11.4 & $4 d 1 u(2)$ & 3.9 \\
\hline $3 p 1 u(2)$ & 11.1 & $4 d 2 g(2)$ & 2.6 \\
\hline \multirow[t]{5}{*}{$4 s(1)$} & 9.1 & $4 d 2 u(2)$ & 2.8 \\
\hline & & $5 s(2)$ & 19.3 \\
\hline & & $5 p 0(2)$ & 18.9 \\
\hline & & $5 p 1 g(2)$ & 7.3 \\
\hline & & $5 p 1 u(1)$ & 7.4 \\
\hline Total $(\mathrm{eV})$ & 169.6 & Total $(\mathrm{eV})$ & 158.8 \\
\hline \multicolumn{2}{|c|}{$80-\mathrm{keV} \mathrm{He}^{+} \rightarrow{ }^{37} \mathrm{Rb}$} & \multicolumn{2}{|c|}{$80-\mathrm{keV} \mathrm{He}^{+} \rightarrow{ }^{53} \mathrm{I}$} \\
\hline Shell & $\begin{array}{c}\text { Energy loss } \\
\text { (eV/electron) }\end{array}$ & Shell & $\begin{array}{c}\text { Energy loss } \\
\text { (eV/electron) }\end{array}$ \\
\hline $3 s(2)$ & 0.7 & $4 s(2)$ & 3.7 \\
\hline $3 p 0(2)$ & 6.5 & $4 p 0(2)$ & 4.1 \\
\hline $3 p 1 g(2)$ & 1.5 & $4 p 1 g(2)$ & 1.9 \\
\hline $3 p 1 u(2)$ & 1.5 & $4 p 1 u(2)$ & 1.1 \\
\hline $3 d 0(2)$ & 10.5 & $4 d 0(2)$ & 17.8 \\
\hline $3 d 1 g(2)$ & 1.2 & $4 d 1 g(2)$ & 5.5 \\
\hline $3 d 1 u$ (2) & 0.6 & $4 d 1 u$ & 5.0 \\
\hline $3 d 2 g(2)$ & 0.5 & $4 \mathrm{~d} 2 \mathrm{~g}(2)$ & 3.0 \\
\hline $3 d 2 u(2)$ & 0.4 & $4 d 2 u(2)$ & 3.5 \\
\hline $4 s(2)$ & 20.5 & $5 s(2)$ & 18.9 \\
\hline $4 p 0(2)$ & 26.4 & $5 p 0(2)$ & 19.7 \\
\hline $4 p 1 g(2)$ & 9.3 & $5 p 1 g(2)$ & 7.5 \\
\hline $4 p 1 u(2)$ & 9.2 & $5 p 1 u(1)$ & 7.6 \\
\hline $5 s(1)$ & 8.3 & & \\
\hline Total (eV) & 185.8 & Total $(\mathrm{eV})$ & 187.0 \\
\hline
\end{tabular}

As mentioned above, the energy losses due to the skimming effect $\langle\Delta E\rangle / 2 R(35 \mathrm{eV} / \AA$ for $\mathrm{RbI}$ and $30 \mathrm{eV} / \AA$ for $\mathrm{KI})$ are much larger than the Ziegler's stopping power values of $12 \mathrm{eV} / \AA$ for $\mathrm{RbI}$ and $10.5 \mathrm{eV} / \AA$ for KI [2]. Concerning the random energy loss $S_{e}$, the roughly estimated values of $S_{e}^{\mathrm{RbI}}=16$ and $S_{e}^{\mathrm{KI}}=14 \mathrm{eV} / \AA$, respectively, for 80 - and 70-keV He${ }^{+}$passing through $\mathrm{RbI}$ and $\mathrm{KI}$, respectively, are also considerably larger than the Ziegler's stopping powers. There is, of course, an energy uncertainty coming from the incoming and outgoing energy difference. However, the outgoing energy is above $90 \%$ of the incoming energy for the present scattering geometries. Therefore, this energy uncertainty contributing to random energy loss is $5 \%$ at most. In the case of the local energy loss, the contribution to the energy loss uncertainty is less than $10 \%$, comparable with the errors estimated from the uncertainties mainly originating from the deconvolution 
TABLE III. Local (skimming effect) and random energy loss values determined for 80 - and $70-\mathrm{keV} \mathrm{He}^{+}$ions incident on $\mathrm{RbI}(001)$ and KI(001) by MEIS spectrum analysis, which are compared with theoretical values calculated from the coupled-channel method [9] and with semiempirical random energy loss given by Ziegler [2].

\begin{tabular}{|c|c|c|c|c|}
\hline & \multicolumn{2}{|c|}{$80-\mathrm{keV} \mathrm{He}^{+} \rightarrow \mathrm{RbI}(001)$} & \multicolumn{2}{|c|}{ 70-keV He${ }^{+} \rightarrow \mathrm{KI}(001)$} \\
\hline & $\begin{array}{l}\text { Local energy } \\
\text { loss }(\mathrm{eV})\end{array}$ & $\begin{array}{l}\text { Random energy } \\
\text { loss }(\mathrm{eV} / \AA)\end{array}$ & $\begin{array}{l}\text { Local energy } \\
\text { loss }(\mathrm{eV})\end{array}$ & $\begin{array}{c}\text { Random energy } \\
\text { loss }(\mathrm{eV} / \AA)\end{array}$ \\
\hline MEIS & $\Delta E(\mathrm{I})=187 \pm 20$ & $S_{e}^{\mathrm{RbI}}=16 \pm 1$ & $\Delta E(\mathrm{I})=165 \pm 15$ & $S_{e}^{\mathrm{KI}}=14 \pm 1$ \\
\hline Observation & $\Delta E(\mathrm{Rb})=174 \pm 20$ & & $\Delta E(\mathrm{~K})=120 \pm 20$ & \\
\hline Theory & $\Delta E(\mathrm{I})=187.0$ & & $\Delta E(\mathrm{I})=158.8$ & \\
\hline Coupled channel & $\Delta E(\mathrm{Rb})=185.8$ & & $\Delta E(\mathrm{~K})=169.6$ & \\
\hline Ziegler's random energy loss & & $S_{e}^{\mathrm{RbI}}=12.0$ & & $S_{e}^{\mathrm{KI}}=10.5$ \\
\hline
\end{tabular}

procedure. Note that the Ziegler's random energy loss formula acts as a useful guide but does not always give reliable data in the low- and medium-energy regimes.

\section{CONCLUSION}

The energy loss of ions passing close to a lattice-site atom before and after a large-angle collision is strongly enhanced, due to the so-called skimming effect. This phenomenon is quite different from the energy losses of ions penetrating in a random or channeling direction, because the ion impinging and/or emerging along a major crystal axis before or after a large-angle collision takes a path close to a lattice-site atom located in the crystal axis and thus tends to excite the inner shell electrons. The excellent energy resolution of the toroidal ESA makes it possible to resolve energetically each scattering component from the top- and second-layer atoms. The resulting energy difference between the two scattering components originates from two parts: (i) local energy loss subjected by skimming through a lattice-site atom, and (ii) usual random energy loss proportional to a path length. We measured the MEIS spectra using 70- and $80-\mathrm{keV} \mathrm{He}^{+}$ions for $\mathrm{KI}(001)$ and $\mathrm{RbI}(001)$ substrates under various kinds of scattering geometries and decomposed the surface peaks into basically three scattering components from the top-, second-, and third-layer atoms considering the hitting probabilities, which were calculated from the MC simulations of $\mathrm{He}$ ion trajectories taking account of enhanced and correlated thermal vibrations. The local energy losses were determined to be $\Delta E(\mathrm{I})=187 \pm 20$ and $\Delta E(\mathrm{Rb})=174 \pm 20 \mathrm{eV}$ for $80-\mathrm{keV} \mathrm{He}^{+}$incident on $\mathrm{RbI}(001)$ and $\Delta E(\mathrm{~K})=120 \pm 20$ and $\Delta E(\mathrm{I})=165 \pm 10 \mathrm{eV}$ for $70-\mathrm{keV} \mathrm{He}^{+}$incident on $\mathrm{KI}(001)$. The above local energy losses $\langle\Delta E\rangle / 2 R$ are much larger than the Ziegler's random stopping powers by more than three times. Simultaneously, the random energy losses for 80- and
70-keV $\mathrm{He}^{+}$passing through $\mathrm{RbI}$ and $\mathrm{KI}$, respectively, are estimated to be $16 \pm 1$ and $14 \pm 1 \mathrm{eV} / \AA$, which are also considerably larger than the Ziegler's energy loss values of 12.0 and $10.5 \mathrm{eV} / \AA$. This may be due to either additional excitation channels at the very surfaces or inaccuracies of the Ziegler's stopping procedure at low and medium energies. The local energy losses determined experimentally for skimming through a $\mathrm{Rb}$ and an $\mathrm{I}$ atom of $\mathrm{RbI}(001)$ and for passing close to an I atom of $\mathrm{KI}(001)$ are in good agreement with the calculated ones by the coupled-channel method, while for a $\mathrm{K}$ atom of $\mathrm{KI}(001)$ the observed local energy loss deviates significantly from the coupled-channel calculations by almost $30 \%$. This deviation may be attributed to use of the independent particle model, where the effect of dynamical screening is not taken into account, which may be important for slow, heavy projectiles. Despite that, the local energy losses obtained by the MEIS spectrum analysis are basically consistent with the impact-parameter-dependent energy losses given by the coupled-channel calculations.

Such a strongly enhanced energy loss works effectively to resolve each scattering component from the top-, second-, and third-layer atoms in MEIS spectra. However, we must note that surface peaks should be deconvoluted carefully considering the hitting probabilities for the atoms of each layer, which can be calculated from MC simulations of ion trajectories. It is emphasized that such a strongly enhanced electronic energy loss is a key issue to analyze exactly the MEIS spectra from subsurface ordered structures.

\section{ACKNOWLEDGMENTS}

The authors would like to thank A. Visikovskiy for his useful comments and support in the MEIS measurement. This work was partly supported by the Academic Frontier Project of the Ministry of Education, Japan.
[1] H. H. Andersen and J. F. Ziegler, Hydrogen Stopping Powers and Ranges in All Elements (Pergamon, New York, 1977).

[2] J. F. Ziegler, Helium Stopping Powers and Ranges in All Elements (Pergamon, New York, 1977).

[3] J. F. Ziegler, J. P. Biersack, and W. Littmark, The Stopping and Range of Ions in Matter (Pergamon, New York, 1985).

[4] D. S. Gemmel, Rev. Mod. Phys. 46, 129 (1974).
[5] P. J. M. Smulders and D. O. Boerma, Nucl. Instrum. Methods B 29, 471 (1987).

[6] Y. Kido, A. Ikeda, Y. Yamamoto, J. Nakata, H. Yamaguchi, and K. Takahei, Phys. Rev. B 49, 14387 (1994).

[7] A. Hentz, G. S. Parkinson, P. D. Quinn, M. A. Muñoz-Márquez, D. P. Woodruff, P. L. Grande, G. Schiwietz, P. Bailey, and T. C. Q. Noakes, Phys. Rev. Lett. 102, 096103 (2009). 
[8] Y. Kido, T. Nishimura, Y. Hoshino, and H. Namba, Nucl. Instrum. Methods B 161-163, 371 (2000).

[9] G. Schiwietz, Phys. Rev. A 42, 296 (1990).

[10] P. L. Grande and G. Schiwietz, Nucl. Instrum. Methods B 132, 264 (1997).

[11] Y. Kido and T. Okazawa, Surf. Rev. Lett. 10, 389 (2003).

[12] P. L. Grande, A. Hentz, R. P. Pezzi, I. J. R. Baumvol, and G. Schiwietz, Nucl. Instrum. Methods B 256, 92 (2007).

[13] M. Hazama, Y. Kitsudo, T. Nishimura, Y. Hoshino, P. L. Grande, G. Schiwietz, and Y. Kido, Phys. Rev. B 78, 193402 (2008).

[14] J. Lindhard and M. Scharff, Mat. Fys. Medd. K. Dan. Vidensk. Selsk. 27, 15 (1953).

[15] Y. Kido and T. Koshikawa, Phys. Rev. A 44, 1759 (1991).
[16] H. Matsumoto, K. Mitsuhara, A. Visikovskiy, T. Akita, N. Toshima, and Y. Kido, Nucl. Instrum. Methods B 268, 2281 (2010).

[17] K. Mitsuhara, H. Okumura, T. Matsuda, M. Tagami, A. Visikovskiy, and Y. Kido, Nucl. Instrum. Methods B 276, 56 (2012).

[18] T. Okazawa, S. Ohno, Y. Hoshino, T. Nishimura, and Y. Kido, Nucl. Instrum. Methods B 183, 108 (2001).

[19] T. Okazawa, T. Nishimura, and Y. Kido, Phys. Rev. B 66, 125402 (2002).

[20] C. R. A. Catlow, K. M. Diller, and M. J. Norgett, J. Phys. C 10, 1395 (1977).

[21] J. F. van der Veen, Surf. Sci. Rep. 5, 199 (1985).

[22] D. P. Jackson and J. H. Barrett, Comput. Phys. Commun. 13, 157 (1977). 